\title{
BMJ Open Conservative and surgical modalities in the management of paediatric parapneumonic effusion and empyema: a protocol for a living systematic review and network meta-analysis
}

\author{
Emily Allin (D) , ${ }^{1,2}$ Nassr Nama (D) , ${ }^{1,2}$ Michael A Irvine (D) , ${ }^{3}$ Colleen Pawliuk, ${ }^{3}$ \\ Marie Wright, ${ }^{1,4}$ Matthew Carwana ${ }^{1,3,5}$
}

To cite: Allin E, Nama N, Irvine MA, et al. Conservative and surgical modalities in the management of paediatric parapneumonic effusion and empyema: a protocol for a living systematic review and network meta-analysis. BMJ Open 2021;11:e045010. doi:10.1136/ bmjopen-2020-045010

- Prepublication history and additional material for this paper are available online. To view these files, please visit the journal online (http://dx.doi. org/10.1136/bmjopen-2020045010).

Received 19 September 2020 Revised 09 February 2021 Accepted 28 February 2021

Check for updates

(c) Author(s) (or their employer(s)) 2021. Re-use permitted under CC BY-NC. No commercial re-use. See rights and permissions. Published by BMJ.

For numbered affiliations see end of article.

\section{Correspondence to}

Dr Nassr Nama, Pediatrics, BC Children's Hospital, Vancouver, BC, Canada;

nassr.nama@alumni.ubc.ca

\section{ABSTRACT}

Introduction Parapneumonic effusion and empyema are common complications of paediatric pneumonia. Acceptable treatment modalities for large parapneumonic effusions include antibiotics alone or in conjunction with surgical interventions. Clear guidelines on the best treatment approach are lacking and mostly based on evidence prior to widespread pneumococcal conjugate 13-valent vaccination (PCV-13).

Methods and analysis A living systematic review and network meta-analysis will be performed comparing the five treatment modalities: (1) antibiotics alone; (2) chest tube drainage without fibrinolytics; (3) chest tube drainage with fibrinolytics; (4) video-assisted thoracoscopic surgery and (5) open thoracotomy. The review protocol is reported following the Preferred Reporting Items for Systematic Review and Meta-Analysis Protocols guidelines. Eligible studies are randomised controlled trials comparing any pair of interventions in paediatric patients with empyema or parapneumonic effusion. The following databases will be searched: Ovid MEDLINE, EMBASE, Cochrane Central Register of Controlled Trials (CENTRAL), Web of Science, LILACS and Google Scholar. Citation screening and data extraction will be completed using a validated crowdsourcing methodology using InsightScope. To assess the risk of bias, we will use the revised Cochrane risk of bias tool for randomised trials. The primary outcome of the study is the length of stay. Secondary outcomes are (1) periprocedural complications and (2) need for re-intervention. A frequentist network meta-analysis design will be implemented with a random-effects model comparing different interventions. In a subgroup analysis, studies and patients will be stratified by the size of pleural effusion and the date of trial (pre/post-PCV-13). Eligible citations and available results will be uploaded to an online database, hosted on Open Science Framework. The database will be updated at least every 4 months with any newly published research.

Ethics and dissemination No ethics review is required for this study. Results will be published in a peer-reviewed journal. Data will be available as part of an online database summarising the evidence of this living systematic review. PROSPERO registration Pending peer review.
Strengths and limitations of this study

- This systematic review will compare different intervention modalities in the treatment of paediatric empyema.

- Network meta-analysis will allow us to synthesise direct and indirect data to generate a comprehensive comparison of each intervention pair, including those not studied in a head-to-head trial.

- This living review will maintain an up-to-date summary of evidence in the field and incorporate any ongoing trials.

- Crowdsourcing steps of the systematic review process will facilitate the broadening of the search strategy and reduce the time to incorporate primary literature into the living review.

- As published trials in the field are small, the quality of the generated summary of evidence might be low.

\section{BACKGROUND}

Pneumonia continues to be the leading cause of morbidity and mortality in the paediatric population globally. ${ }^{1}$ The introduction of widespread pneumococcal conjugate 13-valent vaccination (PCV-13) programmes has led to a significant reduction in the incidence and mortality of community-acquired pneumonia (CAP). ${ }^{2} 3$ However, this disease's burden remains high, accounting for $15 \%$ of deaths in young children worldwide. ${ }^{4}$ Approximately $12 \%$ of cases progress to severe illness, with parapneumonic effusion and pleural empyema being the most common complications. ${ }^{5}$ For simplicity, this paper will use the term 'effusion' to refer to parapneumonic effusion and pleural empyema.

The presence of effusion leads to a prolonged length of stay (LOS), escalation of care, morbidity and increased healthcare resources utilisation. ${ }^{6-10}$ Management of the 
effusion is controversial. ${ }^{67}$ Accepted treatment modalities include antibiotics alone, chest tube drainage with or without fibrinolytics, video-assisted thoracoscopic surgery (VATS) and open thoracotomy (OT) with decortication. In general, guidelines around complicated pneumonia management agree that small and most moderate-sized effusions can be managed with antibiotics alone. ${ }^{9-13}$ Meanwhile, drainage is suggested for large effusions and effusions associated with a high degree of respiratory distress. Despite these guidelines, there is significant variability in clinical practice among physicians in managing parapneumonic effusions and empyema in children. ${ }^{14-16}$

Additionally, published guidelines are mostly based on pre-PCV-13 studies. The global introduction of PCV-7 and PCV-13 has led to significant changes in paediatric pneumonia's clinical presentation. While the overall incidence of CAP has decreased in the past couple of decades, ${ }^{17}$ there was a notable increase in the incidence of empyema post-PCV-7. ${ }^{17-20}$ With the emergence of pneumococcal strains not included in the vaccine, there have been significant changes in the epidemiology and pathogenesis of CAP post-PCV-13. Given these changes, the current applicability of these guidelines is unclear.

To summarise currently available evidence, we will systematically review the existing literature for management of parapneumonic effusion and empyema. The primary aim of this systematic review is to identify which of antibiotics alone, chest tube drainage with/without fibrinolytics, VATS or OT leads to a shortened LOS in children with empyema. These five treatment arms were selected as they are well documented in the literature and are recommended therapies by guidelines. Given the heterogeneity of empyema staging, it is essential to compare outcomes between comparable group of patients. Limiting the systematic review to randomised controlled trials (RCTs) would minimise the potential for confounding. Furthermore, a subgroup analysis will allow us to stratify patients by the size of the stage of parapneumonic effusion and the date of trial (pre/post-PCV-13). As this is an active research field with multiple ongoing studies, ${ }^{21}{ }^{22}$ we will undertake a living systematic review methodology. This will allow us to update our review findings with new evidence regularly. The network meta-analysis (NMA) will enable us to synthesise direct and indirect data to generate a comprehensive comparison of all possible pairwise comparisons available in the network.

\section{METHODS AND ANALYSIS}

The protocol of this study is reported following the Preferred Reporting Items for Systematic Review and Meta-Analysis Protocols (PRISMA-P) guidelines (online supplemental file 1), ${ }^{23}$ and will be prospectively registered on PROSPERO (pending peer review). The final review will be reported in accordance with the PRISMA extension for NMA. ${ }^{24}$

\section{Search strategy and selection criteria}

The search is aimed to identify all citations relevant to empyema/parapneumonic effusion AND pediatric/children AND randomized controlled trials AND intervention/treatment/management/surgery/efficacy, with no filtering of search by language or date. Cochrane search filter was used to identify RCTs. ${ }^{25} \mathrm{~A}$ draft Ovid MEDLINE search strategy is provided in online supplemental file 2. This will be peer reviewed by another librarian using the PRESS Evidence-Based Checklist, and then translated to the other databases. ${ }^{26}$ The following databases will be searched: Ovid MEDLINE, EMBASE, Cochrane Central Register of Controlled Trials (CENTRAL), Web of Science Core Collection, LILACS and Google Scholar. In addition, a comprehensive grey literature search will be undertaken: clinicaltrials.gov, WHO International Clinical Trials Registry Platform, Papers First (via WorldCat), Proceedings (via WorldCat), Networked Digital Library of Theses and Dissertations, ProQuest Dissertations and Theses Global and OAister (narrowing to theses/dissertations; via WorldCat). The reference list of any included study or relevant systematic review will be searched for other potentially eligible studies.

\section{Eligibility criteria}

Studies will be included if they are RCTs and targeted patients under 18 years of age with parapneumonic effusion or pleural empyema as complications of pneumonia. Parapneumonic effusion is defined as any collection of pleural fluid associated with an underlying pneumonia. ${ }^{27}$ Empyema is defined as the presence of grossly purulent pleural fluid. We will retain studies in English, French, Arabic, Chinese, German, Italian, Polish, Portuguese, Russian or Spanish. Trials must include at least one of the following therapeutic interventions:

1. Antibiotics alone.

2. Chest tube drainage without fibrinolytics.

3. Chest tube drainage with fibrinolytics.

4. VATS.

5. OT.

The interventions mentioned above are restricted to primary therapy rather than secondary interventions following failed treatments (eg, salvage VATS following a failed chest tube drainage). Studies that included only patients who experienced failure of primary therapy will be excluded. There is no restriction on antibiotic or fibrinolytic therapy's nature/details, such as the type of antibiotic/fibrinolytic, dosage or frequency, compliance level or the treatment setting. No restriction is applied on the presented outcomes. Studies will be excluded if they did not have any patients below 18 years of age, or were limited to the following populations: chronic lung diseases (including cystic fibrosis and ciliary dyskinesia), pre-existing cardiac or neurological disease, immunodeficiency, malignant effusions, post-cancer chemotherapy or children receiving home mechanical ventilation. If a trial included eligible patients under 18 years of age as 
well as adults, results would be included if the data for the eligible age group were presented separately.

\section{Crowdsourcing of study selection}

Citation screening will be completed on InsightScope, a platform designed for crowdsourcing systematic reviews. ${ }^{28}$ A recruitment email will be sent out to registered crowd members and disseminated through paediatric interest groups, research interest groups, undergraduate societies across Canada and the Cochrane task exchange platform. The crowd is mostly comprised of students with postsecondary training (undergraduate/medical/graduate students). ${ }^{29} 30$

Two of the expert reviewers will screen $10 \%$ of citations. A draft of screening criteria is prepared as part of this protocol (online supplemental file 3). Screening criteria will be reviewed and amended if there is a disagreement between the two experts. From this set of citations, a qualification set of randomly selected 50 citations (containing at least five eligible ones) will be used as the reference standard to which the crowd members are compared. Crowd members will need to demonstrate acceptable performance with citation screening on the qualification set (sensitivity $\geq 80 \%$ and specificity $\geq 70 \%$ ). Reviewers with sensitivity between $80 \%$ and $90 \%$ will receive further training before participating in the full review.

To improve sensitivity, each abstract will be screened independently in triplicate, with an expert resolving conflicts. ${ }^{30}$ At the full-text stage, the crowd will upload the full-text articles and screen the full citations similarly. The final list of retained citations by the crowd will be screened by an expert to confirm eligibility.

\section{Data extraction}

A draft data extraction form was prepared as part of this protocol (online supplemental file 4). This will be piloted by two independent experts on five eligible citations. Conflicts will be discussed and the crowd will use a refined extraction form. Data will be extracted independently by two crowd members and verified by one of the expert investigators. The extraction form includes the following: (1) study identification; (2) methodological characteristics and (3) risk of bias assessment (as detailed below). The following data are to be collected separately for patients who received one of the four treatment arms: (1) description of the population; (2) completed investigations; (3) treatment course (details on antibiotic management as well as the intervention of interest) and (4) outcomes (as detailed below).

Corresponding authors of eligible studies will be contacted to obtain any missing data and confirm whether they are aware of any additional published or ongoing trials.

\section{Outcomes}

The primary outcome of the study is the LOS. Secondary outcomes defined ad hoc are (1) periprocedural complications and (2) need for re-intervention. If a study presented other outcomes, these will be assessed in an exploratory analysis. For periprocedural complications, we will include pain, bleeding, secondary infections or any other treatment-related adverse events reported by the investigative teams.

\section{Risk of bias assessment}

To assess the risk of bias, we will use the revised Cochrane risk of bias tool for randomised trials. ${ }^{31}$ Each citation will be independently assessed by two crowd members and conflicts resolved by an expert investigator.

\section{Network meta-analysis}

The five treatment arms described above will be compared in an NMA. We will evaluate all eligible RCTs involving any head-to-head comparisons. We will not differentiate based on the type of fibrinolytic. If a sufficient number of trials compared different types of fibrinolytics, this will be explored further in a secondary analysis. A network diagram will be generated and modelled within a frequentist NMA design. A random-effects model will be implemented to account for potential heterogeneity between study populations. A mean difference will be used in the meta-analysis for combining continuous data such as LOS, and OR will be used for binary outcomes such as incidence of periprocedural complications and need for re-intervention. If there are no multiarm trials, we will use meta-regression to synthesise direct and indirect evidence. In the case of multiarm trials, we will use the approach of multivariate meta-analysis. We will estimate rankings of interventions using ranking probability, calculated based on the joint distribution of relative efficacy estimates.

Assumptions made in NMA include homogeneity, similarity and consistency. Studies will be assessed for consistency of design, and influence analysis will be performed to evaluate the assumption of homogeneity. Heterogeneity of treatment effects will be explored visually using a forest plot and statistically using $\mathrm{I}^{2}$ based on the Cochran's $Q$ test. We will compare data generated via the random-effects model with a fixed-effect model in a sensitivity analysis.

Results from direct and indirect comparisons of the same two treatment interventions will be reviewed for consistency. Consistency will be addressed using node splitting via a network meta-regression model. Node splitting will assess that the relative effect of two treatments based on direct comparisons is comparable with the same effect based on indirect comparisons. Assessment of transitivity will be carried out by comparing the distribution of effect modifiers across the different comparisons. Assessment of incoherence will be evaluated with the Separating Indirect from Direct Evidence technique. This would entail obtaining the statistical incoherence factor by measuring the absolute difference between direct and indirect summary estimates for the pairwise comparisons. The potential for publication bias will be investigated visually using a funnel plot. 
Grades of Recommendation, Assessment, Development and Evaluation will be used to evaluate the overall quality of evidence, through consideration of five domains: risk of bias, inconsistency, indirectness, imprecision and publication bias. These provide an overall quality of the evidence rated as high, moderate, low or very low. ${ }^{32}$ We will use CINeMA to assess the confidence in NMA (http://cinema.ispm.ch/).

\section{Data analysis}

Data analysis will be conducted in $\mathrm{R}$ (V.4.0.0). In a subgroup analysis, studies and patients will be stratified by the stage of parapneumonic effusion and the date of trial (pre/post-PCV-13). Parapneumonic effusions and empyema will be classified based on the American College of Chest Physicians staging system ${ }^{2733}$ :

- Stage I: minimal uncomplicated parapneumonic effusion $(<10 \mathrm{~mm}$ on lateral decubitus).

- Stage II: small-to-moderate uncomplicated parapneumonic effusion $(>10 \mathrm{~mm}$ and less than half of the hemithorax).

- Stage III: complicated parapneumonic effusion (large effusion greater than half of the hemithorax, loculated effusion, or effusion with a positive gram stain or culture).

- Stage IV: empyema (grossly purulent effusion).

We will use 2010 as a cut-off to distinguish between prePCV-13 and post-PCV-13 studies. ${ }^{34}$ Studies that span both periods will be excluded from the subgroup analysis.

\section{Living systematic review}

Eligible citations and available results will be uploaded to an online database, hosted on Open Science Framework. New citations will be screened and extracted, similar to the original review. The database will be updated at least every 4 months with any newly published research. The synthesis of available evidence will also be updated to reflect the most up-to-date estimates of the measured outcomes.

\section{Patient and public involvement}

No patients or public will be involved in this systematic review.

\section{Author affiliations}

${ }^{1}$ Faculty of Medicine, The University of British Columbia, Vancouver, British Columbia, Canada

${ }^{2}$ Pediatrics, British Columbia Children's Hospital, Vancouver, British Columbia, Canada

${ }^{3}$ Evidence to Innovation, British Columbia Children's Hospital Research Institute, Vancouver, British Columbia, Canada

${ }^{4}$ Division of Respiratory Medicine, British Columbia Children's Hospital, Vancouver, British Columbia, Canada

${ }^{5}$ Division of General Pediatrics, British Columbia Children's Hospital, Vancouver, British Columbia, Canada

Contributors All authors conceptualised and designed the protocol, critically revised the manuscript, approved the final draft and agreed to be accountable for all aspects of the work. In addition, EA drafted the initial manuscript and developed the planned data collection and analysis; NN developed the living systematic review process and data analysis; MI designed the network meta-analysis; CP designed the search strategy; and MW and MC provided content expertise and supervised the project.

Funding The authors have not declared a specific grant for this research from any funding agency in the public, commercial or not-for-profit sectors.

Competing interests None declared.

Patient consent for publication Not required.

Provenance and peer review Not commissioned; externally peer reviewed.

Supplemental material This content has been supplied by the author(s). It has not been vetted by BMJ Publishing Group Limited (BMJ) and may not have been peer-reviewed. Any opinions or recommendations discussed are solely those of the author(s) and are not endorsed by BMJ. BMJ disclaims all liability and responsibility arising from any reliance placed on the content. Where the content includes any translated material, BMJ does not warrant the accuracy and reliability of the translations (including but not limited to local regulations, clinical guidelines, terminology, drug names and drug dosages), and is not responsible for any error and/or omissions arising from translation and adaptation or otherwise.

Open access This is an open access article distributed in accordance with the Creative Commons Attribution Non Commercial (CC BY-NC 4.0) license, which permits others to distribute, remix, adapt, build upon this work non-commercially, and license their derivative works on different terms, provided the original work is properly cited, appropriate credit is given, any changes made indicated, and the use is non-commercial. See: http://creativecommons.org/licenses/by-nc/4.0/.

\section{ORCID iDs}

Emily Allin http://orcid.org/0000-0003-1401-8503

Nassr Nama http://orcid.org/0000-0002-0199-3599

Michael A Irvine http://orcid.org/0000-0003-4785-8998

\section{REFERENCES}

1 Tramper-Stranders GA. Childhood community-acquired pneumonia: a review of etiology- and antimicrobial treatment studies. Paediatr Respir Rev 2018;26:41-8.

2 le Roux DM, Zar HJ. Community-acquired pneumonia in children - a changing spectrum of disease. Pediatr Radiol 2017;47:1392-8.

3 Zar HJ, Andronikou S, Nicol MP. Advances in the diagnosis of pneumonia in children. BMJ 2017;358:j2739.

4 World Health Organization. Fact sheet - pneumonia, 2019. Available: https://www.who.int/en/news-room/fact-sheets/detail/pneumonia

5 Walker CLF, Rudan I, Liu L, et al. Global burden of childhood pneumonia and diarrhoea. The Lancet 2013;381:1405-16.

6 Messinger Al, Kupfer O, Hurst A, et al. Management of pediatric community-acquired bacterial pneumonia. Pediatr Rev 2017;38:394-409.

7 Chibuk T, Cohen E, Robinson J, et al. Paediatric complicated pneumonia: diagnosis and management of empyema. Paediatr Child Health 2011;16:425-7.

8 Harris M, Clark J, Coote N, et al. British thoracic Society guidelines for the management of community acquired pneumonia in children: update 2011. Thorax 2011;66 Suppl 2:ii1-23.

9 Balfour-Lynn IM, Abrahamson E, Cohen G, et al. Bts guidelines for the management of pleural infection in children. Thorax 2005;60 Suppl 1:i1-21.

10 Erlichman I, Breuer O, Shoseyov D, et al. Complicated community acquired pneumonia in childhood: different types, clinical course, and outcome. Pediatr Pulmonol 2017;52:247-54.

11 Bradley JS, Byington CL, Shah SS, et al. Executive summary: the management of community-acquired pneumonia in infants and children older than 3 months of age: clinical practice guidelines by the pediatric infectious diseases Society and the infectious diseases Society of America. Clinical Infectious Diseases 2011;53:617-30.

12 Proesmans M, De Boeck K. Clinical practice: treatment of childhood empyema. Eur J Pediatr 2009;168:639-45.

13 Islam S, Calkins CM, Goldin AB, et al. The diagnosis and management of empyema in children: a comprehensive review from the apsA outcomes and clinical trials Committee. J Pediatr Surg 2012;47:2101-10.

14 Richards MK, Mcateer JP, Edwards TC, et al. Establishing equipoise: national survey of the treatment of pediatric Para-Pneumonic effusion and empyema. Surg Infect 2017;18:137-42.

15 Hafen GM, Grenzbach A-C, Moeller A, et al. Lack of concordance in parapneumonic effusion management in children in central Europe. Pediatr Pulmonol 2016;51:411-7. 
16 Gold J, Hametz P, Sen Al, et al. Provider knowledge, attitudes, and practices regarding bronchiolitis and pneumonia guidelines. Hosp Pediatr 2019;9:87-91.

17 Ouldali N, Levy C, Minodier P, et al. Long-Term association of 13-Valent pneumococcal conjugate vaccine implementation with rates of community-acquired pneumonia in children. JAMA Pediatr 2019;173:362.

18 Haji T, Byrne A, Kovesi T. Trends in pediatric complicated pneumonia in an Ontario local health integration network. Children 2018;5:36.

19 Principi N, Esposito S. Prevention of community-acquired pneumonia with available pneumococcal vaccines. Int J Mol Sci 2016;18:30.

20 Zampoli M, Kappos A, Wolter N, et al. Etiology and incidence of pleural empyema in South African children. Pediatr Infect Dis $J$ 2015;34:1305-10.

21 Lefevre N. Comparison of intrapleural urokinase and video-assisted Thorascopic surgery in the treatment of parapneumonic pleural effusion in children (UROVATS). Available: https://clinicaltrials.gov/ ct2/show/NCT02165891

22 Baoping X. Efficacy of fibrinolytic agents in complicated pleural effusion. efficacy of fibrinolytic agents in complicated pleural effusion, 2020. Available: https://clinicaltrials.gov/ct2/show/ NCT03716375

23 Moher D, Shamseer L, Clarke M, et al. Preferred reporting items for systematic review and meta-analysis protocols (PRISMA-P) 2015 statement. Syst Rev 2015;4:1.

24 Hutton B, Salanti G, Caldwell DM, et al. The PRISMA extension statement for reporting of systematic reviews incorporating network meta-analyses of health care interventions: checklist and explanations. Ann Intern Med 2015;162:777.
25 Lefebvre C, Glanville J, Briscoe S. Chapter 4: Searching for and selecting studies. In: Higgins JPT, Thomas J, Chandler J, et al, eds. Cochrane Handbook for systematic reviews of interventions. Hoboken: John Wiley \& Sons, 2019: 67-107.

26 McGowan J, Sampson M, Salzwedel DM, et al. PRESS Peer Review of Electronic Search Strategies: 2015 Guideline Statement. J Clin Epidemiol 2016;75:40-6.

27 Heffner JE, Klein JS, Hampson C. Interventional management of pleural infections. Chest 2009;136:1148-59.

28 Nama N, Iliriani K, Xia MY, et al. A pilot validation study of crowdsourcing systematic reviews: update of a searchable database of pediatric clinical trials of high-dose vitamin D. Trans/ Pediatr 2017;6:18-26.

29 Nama N, Sampson M, Barrowman N, et al. Crowdsourcing the citation screening process for systematic reviews: validation study. J Med Internet Res 2019;21:e12953.

30 Nama N, Barrowman N, O'Hearn K, O'Hearn K, et al. Quality control for crowdsourcing citation screening: the importance of assessment number and qualification set size. J Clin Epidemiol 2020;122:160-2.

31 Sterne JAC, Savović J, Page MJ, et al. Rob 2: a revised tool for assessing risk of bias in randomised trials. BMJ 2019;366:14898.

32 Guyatt G, Oxman AD, Akl EA, et al. Grade guidelines: 1. IntroductionGRADE evidence profiles and summary of findings tables. J Clin Epidemiol 2011;64:383-94.

33 Colice GL, Curtis A, Deslauriers J, et al. Medical and surgical treatment of parapneumonic effusions : an evidence-based guideline. Chest 2000;118:1158-71.

34 Geno KA, Gilbert GL, Song JY, et al. Pneumococcal capsules and their types: past, present, and future. Clin Microbiol Rev 2015;28:871-99. 